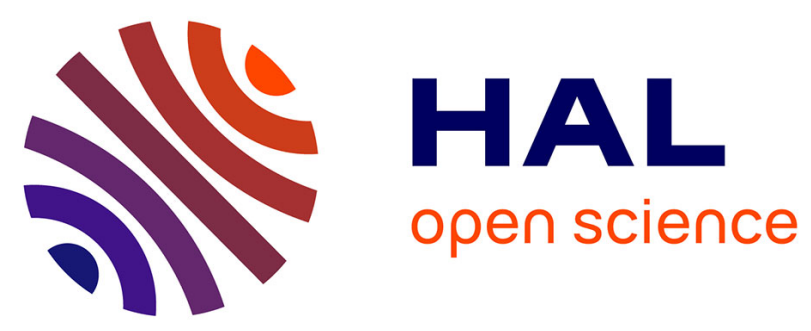

\title{
Réduction de moitié du volume mémoire et de la durée de calcul dans l'usage des algorithmes de transformation de Fourier rapide (FFT)
}

Alain Lifchitz

\section{- To cite this version:}

Alain Lifchitz. Réduction de moitié du volume mémoire et de la durée de calcul dans l'usage des algorithmes de transformation de Fourier rapide (FFT). Acta Crystallographica Section A : Foundations and Advances [2014-..], 1974, 30, pp.86-92. 10.1107/S0567739474000143 . hal-00015151

\section{HAL Id: hal-00015151 https://hal.science/hal-00015151}

Submitted on 23 Feb 2006

HAL is a multi-disciplinary open access archive for the deposit and dissemination of scientific research documents, whether they are published or not. The documents may come from teaching and research institutions in France or abroad, or from public or private research centers.
L'archive ouverte pluridisciplinaire HAL, est destinée au dépôt et à la diffusion de documents scientifiques de niveau recherche, publiés ou non, émanant des établissements d'enseignement et de recherche français ou étrangers, des laboratoires publics ou privés. 
Brrgenau, R. J., Cordes, J., Dolling, G. \& Woods, A. D. B. (1964). Phys. Rev. A 136, 1359-1365.

Chen, S. H. \& Brockhouse, B. N. (1964). Solid State Commun. 2, 73-77.

Chipman, D. R. (1960). J. Appl. Phys. 31, 2012-2015.

CoOPer, M. \& TAYlor, R. I. (1969). Acta Cryst. A25, 714715.

Diana, M., Mazzone, G. \& DeMarco, J. J. (1969). Phys. Rev. 187, 973-979.

Feldman, J. L. (1969). J. Phys. Chem. Solids 30, 367-373. Feldman, J. L. (1970). Phys. Rev. B 1, 448-454.

Herbstein, F. H. \& Smuts, J. (1963). Phil. Mag. 8, 367-385.

Inkinen, O. \& Suortti, P. (1964). Ann. Acad. Sci. Fenn. AVI, 147.
Linkoaho, M. V. (1971). Phil. Mag. 23, 191-198.

Merisalo, M. \& PaAkKari, T. (1973). Mater. Res. Bull. 8, 195-199.

PaAkkari, T., Suortti, P. \& Inkinen, O. (1970). Ann. Acad. Sci. Fenn. AVI, 345.

Sharma, P. K., Semwal, B. S. \& Mehratha, K. N. (1971). $Z$. Naturforsch. 26a, 747-752.

WALFORD, L. K. (1969). Mater. Res. Bull. 4, 137-141.

WALKer, C. B. (1972). PTDS 2. Technical Report, AMMRC, U.S.A.

Walker, C. B. \& Egelstaff, P. A. (1969). Phys. Rev. 177, $1111-1122$.

Wilson, R. H., Skelton, E. F. \& Katz, J. L. (1966). Acta Cryst. 21, 635-638.

Acta Cryst. (1974). A30, 86

\title{
Réduction de Moitié du Volume Mémoire et de la Durée de Calcul dans l'Usage des Algorithmes de Transformation de Fourier Rapide (FFT)
}

\author{
Par Alain Lifchitz \\ Laboratoire de Minéralogie-Cristallographie, associé au C.N.R.S., Université de Paris VI, Tour 16, \\ 4 place Jussieu, 75230 Paris Cedex 05, France
}

(Reçu le 18 juin 1973, accepté le 16 juillet 1973)

\begin{abstract}
After a brief review of the crystallographic Fourier transform problem, this paper compares three methods for its computer implementation with reference to core-storage capacity and computation time: the classic method, the simple Fast Fourier Transform algorithms and a new method. The first method needs, especially for a macromolecular three-dimensional structure, too many operations and therefore too long a computation time and poor accuracy. The remarkable FFT algorithm, on the other hand, leads to large savings in computation time with a better accuracy, but using no crystallographic symmetry wastes too much storage capacity. The third method, using FFT but taking into account Friedel's law by means of a simple in situ process, approximately halves the storage capacity as well as the computation time.
\end{abstract}

\section{Introduction}

Les calculs numériques radiocristallographiques requièrent généralement un stockage mémoire en ordinateur et un temps d'unité centrale notables, en particulier la transformation de Fourier conduite par les méthodes conventionnelles. Le nombre de données à traiter croissant en fonction de la complexité de la structure à étudier, il devient alors crucial de se poser la question de l'optimisation des calculs, notamment lors de l'étude de la structure tridimensionnelle des macromolécules.

L'usage des algorithmes de transformation de Fourier rapide ('Fast Fourier Transform' - FFT), (Cooley \& Tukey, 1965), dans leur forme initiale fait déjà avancer de façon décisive le problème du calcul de la Transformation de Fourier Discrète (TFD), auquel est toujours conduit le cristallographe qui désire effectuer une transformation de Fourier continue. En effet, $K$ étant le nombre de données, une réduction du temps de calcul proportionnelle à $\log _{2} K / K$ par rapport à l'utilisation directe de la définition est obtenue, et cela quelle que soit la dimension de l'espace considéré: 1 pour les corps amorphes, 2 pour le calcul des projections tridimensionnelles, 3 pour lecalcul des densités électroniques. Ce gain en temps de calcul, croissant très rapidement avec $K$, attira évidemment l'attention de certains chercheurs qui en recommandèrent l'emploi en radiocristallographie (Bondot, 1971; Lifchitz, 1971). (Voir l'Appendice pour quelques propriétés utiles de la TFD; le lecteur intéressé par la FFT pourra consulter avec intérêt Cochran et al. (1967) et les nombreux articles qui suivirent dans les revues de traitement du signal et d'informatique surtout aux U.S.A.)

Mais on doit constater que malgré tout la FFT reste encore trop peu utilisée par les cristallographes, peutêtre parce qu'elle nécessite l'utilisation de valeurs numériques complexes et peut ainsi poser des problèmes 
de taille mémoire rapide. Or, comme on le constatera par la suite, il s'avère possible dans la majorité des cas, non seulement de pallier cet inconvénient, mais également d'obtenir un gain sur la durée de calcul de l'ordre de 2 .

\section{Les méthodes de transformation de Fourier en radiocristallographie}

Pour plus de clarté et de simplicité, sans perdre en généralité, nous analyserons en détail le cas unidimensionnel, les cas multidimensionnels n'apportant aucune variation essentielle à la méthodologie. D'autre part, nous nous préoccuperons uniquement dans ce chapitre de la transformation: espace réel $\rightarrow$ espace réciproque; la transformation inverse pouvant être traitée de manière tout-à-fait analogue.

Soit $K=2 N$ un nombre entier pair et $\{X k\}$ la suite de valeurs réelles pour $k=0,1,2, \ldots, K-1$, à transformer. On comparera alors le déroulement de trois modes principaux de calcul de la $\operatorname{TFD}(K)$ de $\{X k\}$, à savoir:

- méthode conventionnelle

- utilisation directe de la $\mathrm{FFT}(K)$

- compactage $+\mathrm{FFT}(K / 2)+$ transformation $\hat{\mathbf{C}}^{-1}$.

Cette comparaison portera d'une part, sur le nombre d'opérations complexes à effectuer, additions et multiplications entre nombres complexes, et, d'autre part, sur le volume de mémoire rapide nécessaire. La Fig. 1 représente l'implantation des valeurs numériques en mémoire, ainsi que différents paramètres caractérisant ces trois méthodes. La représentation a été faite ici pour $K=8 ; R[\hat{Y} k]$ et $I[\hat{Y} k]$ désignent respectivement les parties réelle et imaginaire du nombre complexe $\hat{Y} k$, leurs adresses mémoires sont consécutives conformément au langage Fortran. On remarque qu'une valeur numérique complexe correspondant à la donnée de deux valeurs numériques réelles nécessite un volume mémoire double.

\subsection{Méthode conventionnelle}

Lorsqu'il n'y a pas dispersion anormale, il existe les deux propriétés conjugées:

\begin{tabular}{|c|c|}
\hline Espace réel & Espace réciproque \\
\hline densité électronique réelle & loi de Friedel: \\
\hline & $\begin{array}{l}(N-1) \text { valeurs complexes } \\
\text { indépendantes }+2 \\
\text { valeurs réelles: }\end{array}$ \\
\hline $\begin{array}{l}K \text { valeurs réelles } \\
\text { indépendantes }\end{array}$ & $\begin{array}{l}K \text { valeurs réelles } \\
\text { indépendantes. }\end{array}$ \\
\hline
\end{tabular}

Le calcul est donc limité à un demi-espace réciproque correspondant à $K$ valeurs réelles.

La méthode, qui consiste à appliquer directement la définition de la TFD, implique de conserver $\{X k\}$ en unité centrale jusqu'au calcul du dernier point réciproque. Une utilisation de la mémoire de masse (bande magnétique, disque magnétique, tambour magnétique, etc.) caractérisée par sa lenteur relative, conduirait à des temps de transfert (mémoire de masse $\rightarrow$ unité centrale) cumulés prohibitifs, car il serait nécessaire de transmettre $\{X k\}$ en totalité pour le calcul de chaque point réciproque. Les valeurs calculées $\{\hat{X} q\}$ peuvent elles-mêmes en totalité, s'accumuler en unité centrale [Fig. 1(a), (b)], plus couramment et plus judicieusement aussi, remplir une zone mémoire restreinte, multiple de la taille des enregistrements physiques de la mémoire de masse, servant ainsi de tampon de sortie vers celle-ci. Le gain en volume mémoire rapide peut être de l'ordre de 2 , mais contrairement au cas précédent, le temps de transfert reste raisonnable, un raffinement consistant à utiliser deux tampons en bascule (transfert d'un tampon pendant le remplissage du second), lorsque l'ordinateur permet le mode de transfert asynchrone.

En conclusion, cette méthode classique, bien que très lente, conduit dans la variante avec tampon de sortie à une occupation mémoire proche de l'optimum $K$, et gagne en simplicité de programmation ce qu'elle n'a pas en performance. Elle peut toutefois s'avérer utile pour $K$ petit ou lorsque la totalité du demi-espace réciproque n'est pas nécessaire, mais pour tous les autres cas le grand nombre d'opérations nécessaires conduit à une précision numérique et une durée de calcul peu attrayantes.

\subsection{Utilisation directe des algorithmes $F F T(K)$}

Il existe trois types principaux d'algorithmes FFT: découpage en temps, découpage en fréquence, méthode de Stockham. Les deux premiers, d'efficacité à peu près équivalente en pratique, nécessitent un reclassement des valeurs numériques en mémoire, respectivement en entrée et en sortie. Mais le calcul étant itératif, la TFD vient prendre finalement la place des données, contrairement à la méthode classique, en contribuant ainsi au gain de mémoire. Quant au troisième algorithme et ses variantes, bien que sans reclassement, ils ne permettent pas le calcul in situ, et seront donc rejetés pour ce qui est de notre propos.

Le pouvoir d'accélération de ces algorithmes dépend beaucoup de la décomposition de $K$ en facteurs premiers: $K=2^{p}$ avec $p$ entier donne des résultats proches de l'optimum théorique avec la possibilité d'une programmation aisée; c'est ce choix qui sera fait en pratique. S'il s'avère nécessaire d'avoir $K \neq 2^{p}$, l'astucieux algorithme 'Chirp $z$ - transform' (Rabiner, Schafer \& Rader, 1969) permet de convertir un programme FFT à base 2 en un programme FFT à base arbitraire tout en conservant une excellente efficience.

Mais l'inconvénient majeur des FFT pour le cristallographe, est de porter sur des nombres complexes, ce qui oblige à flanquer les $K$ valeurs réelles envisagées de $K$ valeurs imaginaires nulles [Fig. 1(c)], ... Il en résulte un espace réciproque complet après action de la 


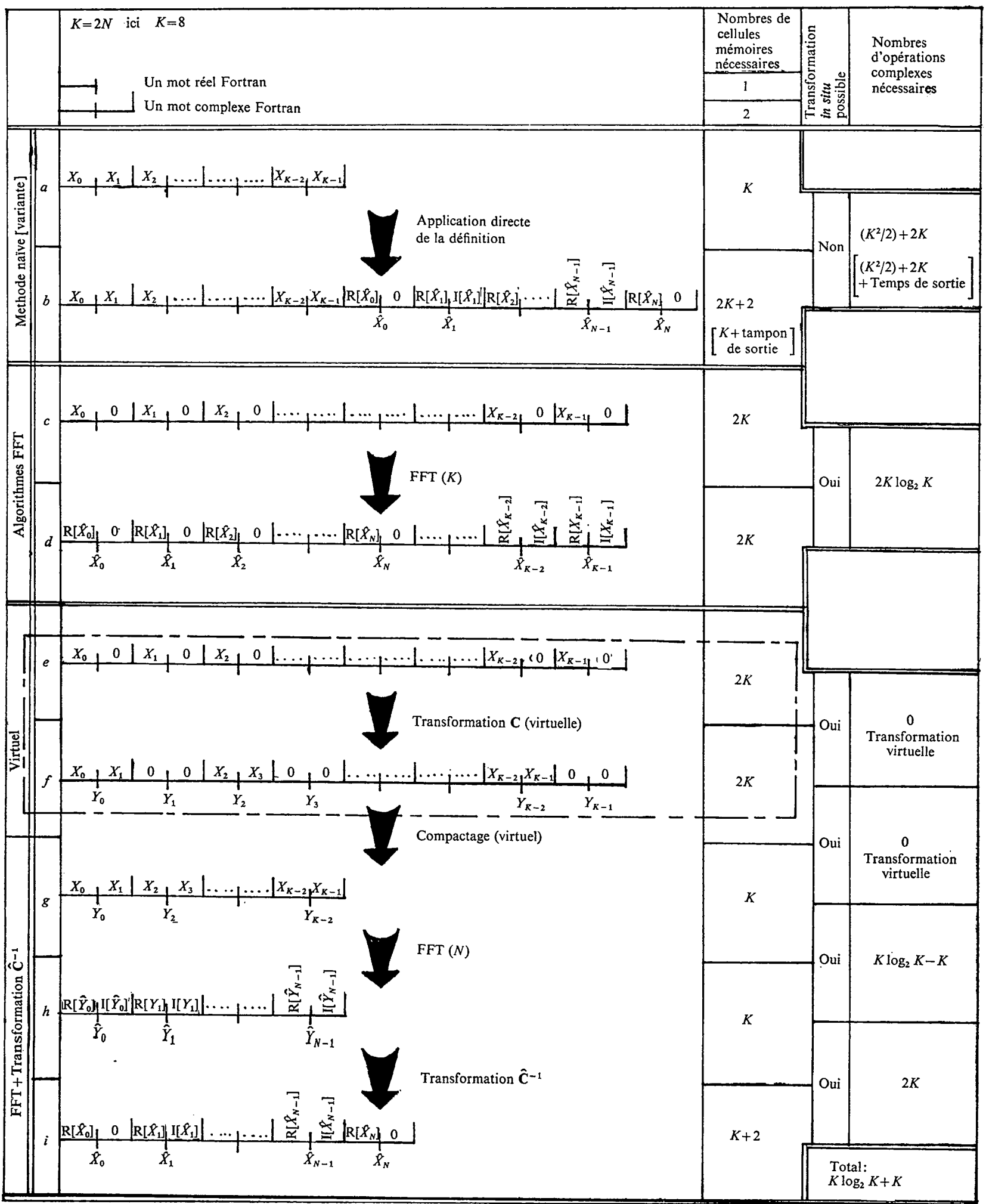

Fig. 1. Les trois méthodes de transformation de Fourier: implantation des données à chaque étape de calcul, nombre d'opérations complexes et nombre de cellules mémoires nécessaires. 
FFT $(K)$ [Fig. $1(d)$ ]. Si la relation de Friedel pouvait intervenir, le volume mémoire et le nombre d'opérations nécessaires seraient réduits de moitié.

La FFT diminue considérablement le nombre d'opérations à effectuer dans l'évaluation d'une TFD, alliant ainsi extrême rapidité et précision contrairement à l'utilisation directe de la relation de définition, mais l'utilisation mémoire ne sera pas optimisée dans la majorité des calculs radiocristallographiques.

\subsection{Compactage suivi de FFT(K/2) et transformation $\hat{\mathbf{C}}^{-1}$}

Il s'agit de concilier les avantages de la FFT et le gain de mémoire possible lorsque la relation de Friedel s'applique. Ceci a été récemment obtenu à une dimension sous certaines conditions restrictives (Vernet, 1971).

Dans ce qui suit, on étudiera une méthode qui, bien que différente, poursuit le même but, et s'utilise quelle que soit la dimension de la TFD. Les $K$ cellules mémoires contenant des valeurs nulles n'existent plus et une FFT(N) suffit à transformer les $K$ données réelles. Son emploi pratique ne sera développé qu'après avoir établi quelques relations indispensables à l'exposé du principe et défini les transformations $\hat{\mathbf{C}}$ et $\mathbf{C}$.

\section{La réduction des calculs}

\subsection{La transformation $\mathbf{C}$}

On considérera tout au long du calcul les $\{X k, l, m\}$ comme K.L.M. données tridimensionnelles réelles dont on se proposera de calculer la TFD:

$$
\{\hat{X} q, r, s\}=\mathbf{T F D}(K, L, M)\{X k, l, m\} .
$$

On forme les ensembles $\{V k, l, m\}$ et $\{W k, l, m\}$ tel que:

$V k, l, m=X k, l, m ; W k, l, m=0 \quad$ si $k$ pair

$V k, l, m=0 \quad ; W k, l, m=X k, l, m$ si $k$ impair,

d'où: $X k, l, m=V k, l, m+W k, l, m$.

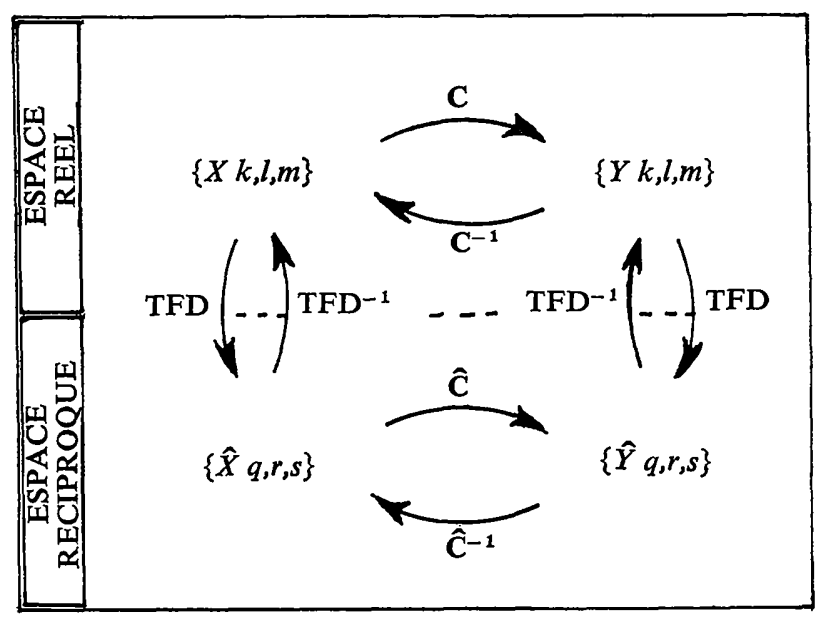

Fig. 2. Les transformations $\mathbf{C}$ et $\widehat{\mathbf{C}}$.
Soit $Y k, l, m=V k, l, m+i W k+1, l, m$ et $\mathbf{C}$ la transformation qui fait passer de $\{X k, l, m\}$ à $\{Y k, l, m\}$.

\subsubsection{Linéaire}

Soit $\{S k, l, m\}$ et $\{T k, l, m\} K . L . M$ données réelles; par la définition même de $\mathbf{C}$ :

$$
\begin{aligned}
& \mathbf{C}\{S k, l, m+\lambda T k, l, m\} \\
& \quad=\mathbf{C}\{S k, l, m\}+\lambda \mathbf{C}\{T k, l, m\} \text { pour tout } \lambda \text { réel } .
\end{aligned}
$$

\subsubsection{C unitaire}

Puisque les $\{X k, l, m\}$ sont réelles, on vérifie immédiatement:

$$
\begin{aligned}
& \sum_{k=0}^{K-1} \sum_{l=0}^{L-1} \sum_{m=0}^{M-1}|Y k, l, m|^{2} \\
&=\sum_{k=0}^{K-1} \sum_{l=0}^{L-1} \sum_{m=0}^{M-1} X^{2} k, l, m \text { si } K \text { pair } .
\end{aligned}
$$

\subsection{La transformation $\hat{\mathbf{C}}$}

$\hat{\mathbf{C}}$ est définie comme la transformation agissant sur $\operatorname{TFD}(K, L, M)\{X k, l, m\}$ pour donner $\operatorname{TFD}(K, L, M)$ $\{Y k, l, m\}$ (Fig. 2).

On constate sur cette Figure que:

$\hat{\mathbf{C}}=\mathbf{T F D} \bigcirc \mathbf{C} \bigcirc \mathbf{T F D}^{-1}$ où ' $\bigcirc$ ' désigne le produit de composition des applications. $\hat{\mathbf{C}}$ est donc unitaire avec C:

$$
\begin{aligned}
& \sum_{q=0}^{K-1} \sum_{r=0}^{L-1} \sum_{s=0}^{M-1}|\hat{X} q, r, s|^{2} \\
& =2 \sum_{q=0}^{N-1} \sum_{r=0}^{L-1} \sum_{s=0}^{M-1}|\hat{Y} q, r, s|^{2} \text { si } K=2 N
\end{aligned}
$$

d'après (7) car $Y 2 k+1, l, m \equiv 0$.

\subsection{Expression de $\hat{\mathbf{C}}$ et $\hat{\mathbf{C}}^{-1}$}

Choisissant $K$ pair en posant $K=2 N$, les propriétés de linéarité (3) et de translation (4) donnent immédiatement:

$$
\begin{gathered}
\hat{X} q, r, s=\hat{V} q, r, s+\hat{W} q, r, s \\
\hat{Y} q, r, s=\hat{V} q, r, s+i \exp (i \pi q / N) \hat{W} q, r, s .
\end{gathered}
$$

On peut donc écrire:

$$
\begin{gathered}
\hat{X}^{*} N-q, L-r, M-s=\hat{V}^{*} N-q, L-r, M-s \\
+\hat{W}^{*} N-q, L-r, M-s \\
\hat{Y}^{*} N-q, L-r, M-s=\hat{V}^{*} N-q, L-r, M-s \\
-i \exp [-i \pi(N-q) / N] \hat{W}^{*} N-q, L-r, M-s .
\end{gathered}
$$

La loi de Friedel (6) s'applique à $\{V k, l, m\}$ et $\{W k, l, m\}$ :

$$
\begin{aligned}
\hat{X}^{*} N-q, L-r, M-s= & \hat{V} N+q, r, s+\hat{W} N+q, r, s \\
\hat{Y}^{*} N-q, L-r, M-s= & \hat{V} N+q, r, s \\
& +i \exp (i \pi q / N) \hat{W} N+q, r, s .
\end{aligned}
$$


Ainsi que les relations de dilatation d'échelle (7) et ( $\left.7^{\prime}\right)$

$$
\begin{aligned}
\hat{X}^{*} N-q, L-r, M-s & =\hat{V} q, r, s-\hat{W} q, r, s \\
\hat{Y}^{*} N-q, L-r, M-s= & \hat{V} q, r, s \\
& -i \exp (i \pi q / N) \hat{W} q, r, s .
\end{aligned}
$$

Eliminer $\hat{V} q, r, s$ et $\hat{W} q, r, s$ entre (12), (13), (12') et (13') permet d'obtenir les relations existant entre $\hat{X} q, r, s$ et $\hat{X}^{*} N-q, L-r, M-s$ d'une part, et $\hat{Y} q, r, s$ et $\hat{Y}^{*} N-q, L-r, M-s$ d'autre part, qui expriment ainsi $\hat{\mathbf{C}}^{-1}$ et $\hat{\mathbf{C}}$.

$$
\hat{\mathbf{C}}^{-1}\left\{\begin{aligned}
\hat{X} q, r, s \quad & {[(\hat{Y} q, r, s} \\
& \left.+\hat{Y}^{*} N-q, L-r, M-s\right) \\
& -i \exp (-i \pi q / N)(\hat{Y} q, r, s \\
& \left.\left.-\hat{Y}^{*} N-q, L-r, M-s\right)\right] / 2 \\
\hat{X}^{*} N-q, L-r, M-s & =[(\hat{Y} q, r, s \\
& \left.+\hat{Y}^{*} N-q, L-r, M-s\right) \\
& +i \exp (-i \pi q / N)(\hat{Y} q, r, s \\
& \left.\left.-\hat{Y}^{*} N-q, L-r, M-s\right)\right] / 2
\end{aligned}\right.
$$$$
\hat{\mathbf{C}}\left\{\begin{aligned}
\hat{Y} q, r, s & =[(\hat{X} q, r, s \\
& \left.+\hat{X}^{*} N-q, L-r, M-s\right) \\
& +i \exp (+i \pi q / N)(\hat{X} q, r, s \\
& \left.\left.-\hat{X}^{*} N-q, L-r, M-s\right)\right] / 2 \\
\hat{Y}^{*}-N q,-L r,-M s & =[(\hat{X} q, r, s \\
& \left.+\hat{X}^{*} N-q, L-r, M-s\right) \\
& -i \exp (+i \pi q / N)(\hat{X} q, r, s \\
& \left.\left.-\hat{X}^{*} N-q, L-r, M-s\right)\right] / 2 .
\end{aligned}\right.
$$

2.3. Applications des transformations $\mathbf{C}, \hat{\mathbf{C}}$ et $\hat{\mathbf{C}}^{-1} \grave{a}$ la réduction des calculs

Imaginons les $K$ éléments de l'espace réel $\{X k\}$ rangés comme pour une $\mathrm{FFT}(K)$ [Fig. $1(e)]$; supposons encore que la transformation $\mathbf{C}$ agisse sur ces $K$ nombres complexes (réels...) pour donner la suite $\{Y k\}$ $k=0,1,2, \ldots, K-1$, et qu'elle soit suivie d'une opération de compactage permettant de ne conserver que les nombres $Y k$ en général non nuls puisque $Y 2 k+1 \equiv 0$ [Fig. $1(g)$ ]. La suite $\{Y 2 k\}$ correspond alors à un stockage des données plus rationnel, identique à celui de la méthode conventionnelle [Fig. 1(a)]. Ces opérations qui ne sont que virtuelles, n'ont pas à être effectuées en réalité, l'entrée en mémoire se faisant directement selon le schéma Fig. $1(\mathrm{~g})$; elles justifient et expliquent néanmoins les étapes ultérieures du calcul. La propriété de dilatation d'échelle (7) permet une FFT $(N)$ pour évaluer $\{\hat{Y} q\}=\mathbf{T F D}(K)\{Y k\}$ en partant de la seule suite $\{Y 2 k\}$ [Fig. 1(h)]. Il suffit alors (Fig. 2) de faire agir la transformation $\hat{\mathbf{C}}^{-1}$ sur les $\{\hat{Y} q\}$ pour obtenir finalement, en utilisant son expression explicite (14), les seuls points réciproques $\hat{X} q$ recherchés, en par- ticulier dans cet exemple un demi-espace réciproque [Fig. $1(i)$ ].

Le volume mémoire utilisé durant les trois étapes réelles du calcul ne dépasse en aucun cas $K+2$, étant ainsi optimum; quant au nombre total d'opérations complexes à effectuer et, par conséquent, au temps de calcul résultant, un bilan s'établit entre un gain dû à l'usage d'une $\mathrm{FFT}(N)$, plutôt que d'une $\mathrm{FFT}(K)$, et une perte impliquée par la nécessité d'une transformation $\hat{\mathbf{C}}^{-1}$ supplémentaire. Comme on pourra le constater sur la Fig. 1, le bilan est largement positif en faveur de la méthode proposée, non seulement comparativement aux méthodes conventionnelles, mais aussi par rapport à l'usage d'une simple $\mathrm{FFT}(K)$.

La méthode proposée permet tout aussi avantageusement de réaliser le passage: espace réciproque $\rightarrow$ espace réel, selon un processus inverse du précédent: transformation $\hat{\mathbf{C}}$ suivie d'une $\mathrm{FFT}^{-1}(N)$ (Fig. 2), fournissant un résultat sous forme compacte.

L'utilisation pratique des groupes de relations (14) et $\left(14^{\prime}\right)$ bénéficie de facteurs favorables dus à leur forme même:

(a) les transformations $\hat{\mathbf{C}}$ et $\hat{\mathbf{C}}^{-1}$ peuvent être menées in situ rendant ainsi effectif le gain en memoire rapide.

(b) Pour les calculs numériques où $q=$ $0,1,2, \ldots,[N / 2],[N / 2]$ désignant la partie entière de $N / 2$, l'usage de chacune des deux relations formant chaque groupe permet de couvrir exhaustivement l'intervalle $q=0,1,2, \ldots, N-1$, en optimisant au maximum les calculs, du fait de leur similitude et de leur symétrie.

(c) (14) et $\left(14^{\prime}\right)$ ne diffèrent que par un simple changement de signe (transformation de $+i$ en $-i$ ): un seul et même sous-programme réalisera les deux transformations $\hat{\mathbf{C}}$ et $\hat{\mathbf{C}}^{-1}$ comme c'est le cas en particulier pour $\operatorname{FFT}(N)$ et $\mathrm{FFT}^{-1}(N)$.

\section{APPENDICE}

\section{Quelques propriétés utiles de la TFD}

\section{Definition et notations}

Etant donné $K . L . M$ échantillons $\{X k, l, m\}$ en général complexes, on définit:

$$
\begin{aligned}
\hat{X} q, r, s=\sum_{k=0}^{K-1} & \sum_{l=0}^{L-1} \sum_{m=0}^{M-1} X k, l, m \\
& \times \exp [-i 2 \pi(k q / K+l r / L+m s / M)]
\end{aligned}
$$

où les $\{\hat{X} q, r, s\}$ désignent les éléments de la TFD à trois dimensions d'ordre $(K, L, M)$ des $\{X k, l, m\}$ qu'on peut noter de manière plus concise:

$$
\{\hat{X} q, r, s\}=\mathbf{T F D}(K, L, M)\{X k, l, m\} .
$$

\section{Périodicités}

$$
\left.\begin{array}{l}
X k+K, l+L, m+M=X k, l, m \\
\hat{X} q+K, r+L, s+M=\hat{X} q, r, s
\end{array}\right\} .
$$


Les deux ensembles de valeurs $\{X k, l, m\}$ et $\{\hat{X} q, r, s\}$ désignent chacun une classe d'équivalence modulo une translation $(\lambda K, \mu L, \nu M)$ avec $\lambda, \mu$ et $\nu$ entiers.

2. Réciprocité

$$
\begin{aligned}
& X k, l, m=\left[\sum_{q=0}^{K-1} \sum_{r=0}^{L-1} \sum_{s=0}^{M-1} \hat{X} q, r, s\right. \\
& \quad \times \exp [+i 2 \pi(k q / K+l r / L+m s / M)]] / K . L . M
\end{aligned}
$$

que l'on notera:

$$
\{X k, l, m\}=\mathbf{T F D}^{-1}(K, L, M)\{\hat{X} q, r, s\} .
$$

\section{Linéarité}

$\operatorname{TFD}(K, L, M)\{X k, l, m+\lambda Y k, l, m\}$

$=\mathbf{T F D}(K, L, M)\{X k, l, m\}+\lambda . \mathbf{T F D}(K, L, M)\{Y k, l, m\}$

avec $\lambda$ quelconque.

\section{Translation}

Soit $t, u$ et $v$, trois nombres entiers définissant une translation, alors:

$$
\begin{aligned}
& \{\hat{X} q, r, s \exp [-i 2 \pi(q t / K+r u / L+s v / M)]\} \\
& =\mathbf{T F D}(K, L, M)\{X k-t, l-u, m-v\} \\
& \{X k, l, m \exp [+i 2 \pi(k t / K+l u / L+m v / M)]\} \\
& \left.=\mathbf{T F D}^{-1}(K, L, M)\{\hat{X} q-t, r-u, s-v\}\right\}
\end{aligned}
$$

\section{Convolution}

Etant donné deux séries $\{X k, l, m\}$ et $\{Y k, l, m\}$, on définit leur produit de convolution périodique par:

$$
\begin{aligned}
\{X k, l, m\} *\{Y k, l, m\}=\{Y k, l, m\} *\{X k, l, m\} \\
=\sum_{h=0}^{K-1} \sum_{i=0}^{L-1} \sum_{j=0}^{M-1} X h, i, j . Y k-h, l-i, m-j .
\end{aligned}
$$

Le théorème de convolution donne alors:

$\operatorname{TFD}(K, L, M)\{X k, l, m\} *\{Y k, l, m\}$

$=\mathbf{T F D}(K, L, M)\{X k, l, m\} . \mathbf{T F D}(K, L, M)\{Y k, l, m\}$. (5)

6. Loi de Friedel

$$
\{X k, l, m\} \text { réels } \Leftrightarrow \hat{X} q, r, s=\hat{X}^{*} K-q, L-r, M-s .
$$

\section{Dilatation d'échelle}

On examinera ici une propriété particulière à la TFD, base de la méthode de réduction des calculs. $K$ sera supposé pair en posant $K=2 N$.

7.0. Supposons que $X k, l, m \equiv 0$ pour $k=1,3, \ldots$, $K-3, K-1$.

$$
\begin{aligned}
\hat{X} q, r, s= & \sum_{k=0}^{K-1} \sum_{l=0}^{L-1} \\
& \sum_{m=0}^{M-1} X k, l, m \\
& \times \exp [-i 2 \pi(k q / K+l r / L+m s / M)] \\
=\sum_{k=0}^{N-1} \sum_{l=0}^{L-1} & \sum_{m=0}^{M-1} X 2 k, l, m \\
& \times \exp [-i 2 \pi(k q / N+l r / L+m s / M)],
\end{aligned}
$$

c'est-à-dire que l'on a la propriété:

$$
\begin{aligned}
& \left\{\begin{array}{l}
K=2 N \\
X 2 k+1, l, m \equiv 0 \Leftrightarrow\{\hat{X} q, r, s\}
\end{array}\right. \\
& =\operatorname{TFD}(N, L, M)\{X 2 k, l, m\} \Leftrightarrow \hat{X} q, r, s=\hat{X} q+N, r, s .
\end{aligned}
$$

7.1. Supposons maintenant que $X k, l, m \equiv 0$ pour $k=0,2, \ldots, K-2, K$

$$
\begin{aligned}
\hat{X} q, r, s=\sum_{k=0}^{K-1} \sum_{l=0}^{L-1} & \sum_{m=0}^{M-1} X k, l, m \\
& \times \exp [-i 2 \pi(k q / K+l r / L+m s / M)] \\
= & \exp (-i 2 \pi q / K) \sum_{k=0}^{N-1} \sum_{l=0}^{L-1} \sum_{m=0}^{M-1} X 2 k+1, l, m \\
& \times \exp [-i 2 \pi(k q / N+l r / L+m s / M)] .
\end{aligned}
$$

On en déduit comme précédemment:

$$
\begin{aligned}
\left\{\begin{array}{l}
K=2 N \\
X 2 k, l, m
\end{array}\right. & \equiv 0 \Leftrightarrow\{\hat{X} q, r, s \exp (+i 2 \pi q / K)\} \\
& =\mathbf{T F D}(N, L, M)\{X 2 k+1, l, m\} \Leftrightarrow \hat{X} q, r, s \\
& =-\hat{X} q+N, r, s
\end{aligned}
$$

8. Conservation de la norme. Théorème de Parseval. Variance

La TFD étant une transformation unitaire conserve le produit scalaire hermitien.

8.0. Conservation du produit scalaire hermitien

$$
\begin{aligned}
\sum_{k=0}^{K-1} \sum_{l=0}^{L-1} \sum_{m=0}^{M-1} X^{*} k, l, m . Y k, l, m \\
=\left[\sum_{q=0}^{K-1} \sum_{r=0}^{L-1} \sum_{s=0}^{M-1} \hat{X}^{*} q, r, s . \hat{Y} q, r, s\right] / K . L . M
\end{aligned}
$$

8.1. Théorème de Parseval. Conservation de l'énergie

$$
\begin{aligned}
& \sum_{k=0}^{K-1} \sum_{l=0}^{L-1} \sum_{m=0}^{M-1}|X k, l, m|^{2} \\
&=\left[\sum_{q=0}^{K-1} \sum_{r=0}^{L-1} \sum_{s=0}^{M-1}|\hat{X} q, r, s|^{2}\right] / \text { K.L.M. }
\end{aligned}
$$


Application particulière de (8) pour $\{Y k, l, m\} \equiv$ et par $\left(8^{\prime}\right)$ : $\{X k, l, m\}$.

8.2. Variance des valeurs de l'espace réel exprimée dans l'espace réciproque

Posons:

Valeur moyenne des $\{X k, l, m\}$

$$
\bar{X}=\left[\sum_{k=0}^{K-1} \sum_{l=0}^{L-1} \sum_{m=0}^{M-1} X k, l, m\right] / K . L . M,
$$

variance des $\{X k, l, m\}$

$$
\sigma^{2}=\left[\sum_{k=0}^{K-1} \sum_{l=0}^{L-1} \sum_{m=0}^{M-1}|X k, l, m-\bar{X}|^{2}\right] / \text { K.L.M. }
$$

Développant l'expression de $\sigma^{2}$ :

$$
\sigma^{2}=\left[\sum_{k=0}^{K-1} \sum_{l=0}^{L-1} \sum_{m=0}^{M-1}|X k, l, m|^{2}\right] / K . L . M-|\bar{X}|^{2}
$$

mais d'après $(0)$ :

$$
\bar{X}=\hat{X} 0,0,0 / K \cdot L \cdot M
$$

$$
\begin{aligned}
\sigma^{2} & =\left[\sum_{k=0}^{K-1} \sum_{l=0}^{L-1} \sum_{m=0}^{M-1}|\hat{X} q, r, s|^{2}-|\hat{X} 0,0,0|^{2}\right] / K^{2} \cdot L^{2} \cdot M^{2} \\
& =\left[\sum_{k=0}^{K-1} \sum_{\substack{l=0 \\
k+l+1}}^{L-1} \sum_{\substack{M=0 \\
M \neq 0}}^{M-1}|\hat{X} q, r, s|^{2}\right] / K^{2} \cdot L^{2} \cdot M^{2} .
\end{aligned}
$$

L'évaluation de la variance $\sigma^{2}$ peut se faire indépendamment de la connaissance de la valeur $\hat{X} 0,0,0$ ainsi que des phases des $\{\hat{X} q, r, s\}$.

\section{References}

Bondot, P. (1971). Acta Cryst. A27, 492-494.

Cochran, W. T., Cooley, W. J., Favin, D. L., Helms, H. D., Kaenel, R. A., Lang, W. W., Maling, G. C., Nelson, D. E., Rader, M. C. \& Welch, P. D. (1967). IEE Trans. Audio Electro-acoustics, AU-15, $\mathrm{n}^{\circ} 2,45-55$. Cooley, W. J. \& Tukey, J. W. (1965). Math. Comput. 19, 297-301.

LIFCHITZ, A. (1971). Sous programme $A B C$ écrit en FORTRAN IV, réalisant l'algorithme de Cooley à 1,2 et 3 dimensions.

RABINER, L. R., SCHAFER, R. W. \& RADER, M. C. (1969). Bell Syst. Techn. J. pp. 1249-1292.

Vernet, J. L. (1971). Proc. IEE, pp. 1531-1532. 